\title{
Quantum Criticality of a Fermi Gas with a Spherical Dispersion Minimum
}

\section{Citation}

Yang, Kun, and Subir Sachdev. 2006. "Quantum Criticality of a Fermi Gas with a Spherical Dispersion Minimum." Physical Review Letters 96 (18). https://doi.org/10.1103/ physrevlett.96.187001.

\section{Permanent link}

http://nrs.harvard.edu/urn-3:HUL.InstRepos:41417306

\section{Terms of Use}

This article was downloaded from Harvard University's DASH repository, and is made available under the terms and conditions applicable to Other Posted Material, as set forth at http:// nrs.harvard.edu/urn-3:HUL.InstRepos:dash.current.terms-of-use\#LAA

\section{Share Your Story}

The Harvard community has made this article openly available.

Please share how this access benefits you. Submit a story.

Accessibility 


\title{
Quantum criticality of a Fermi gas with a spherical dispersion minimum
}

\author{
Kun Yand* and Subir Sachdev \\ Department of Physics, Harvard University, Cambridge, MA 02138, USA
}

(Dated: November 27, 2005)

\begin{abstract}
We describe the quantum phase transition of a Fermi gas occurring when the quasiparticle excitation energy has a minimum in momentum space which crosses zero on a sphere of radius $k_{0} \neq 0$. The quasiparticles have a universal interaction which controls the physical properties in vicinity of the quantum critical point. We discuss possible applications to fermionic superfluids formed by pairing two fermion species, near the point where the densities of the two species become unequal.
\end{abstract}

The study of quantum and thermal fluctuations near second-order, zero temperature quantum phase transitions ("quantum criticality") has been an important theme in modern condensed matter physics [1], providing a unifying framework for many experimental studies of heavy fermion compounds and correlated oxides. With the advent of experiments on trapped, quantum degenerate, ultracold atoms a new arena for the study of tunable quantum phase transitions has opened up; the most prominent example being the study of superfluidinsulator transition of bosonic ${ }^{87} \mathrm{Rb}$ atoms in an optical lattice 2]. Experiments have also studied the condensate formed by pairs of fermionic ${ }^{6} \mathrm{Li}$ atoms with distinct hyperfine states, and very recently these have been extended to a quantum phase transition into a Fermi liquid by unbalancing the density of the hyperfine states [3].

This paper will further explore the physics of paired fermion systems with unequal densities of the two fermion species. Apart from cold atom systems, such problems are also of interest to studies of electronic superconductors in an applied magnetic field [4], and to the formation of paired quark condensates at high nucleon densities [5]. We will introduce and solve a theoretical model of a quantum phase transition that occurs when the densities of the fermion species is initially unbalanced, and describe the universal physical properties in its vicinity. The basis of our analysis will be a second-order quantum critical point (QCP) which allows a systematic renormalization group analysis of arbitrary fermion interactions in its vicinity. We will show that the QCP is a powerful and unifying vantage point for describing a variety of strongly interacting phases obtained as the fermion densities and temperature are varied.

The essential characteristic of our model is that there is a conserved $\mathrm{U}(1)$ "charge", $\mathcal{Q}$, which is carried by the underlying fermions; it is also required that the paired fermion condensate is neutral under this $\mathrm{U}(1)$ symmetry. For the ultracold atom systems, $\mathcal{Q}$ is the difference in the density of the two fermion species, while for electronic superconductors in an applied magnetic field, $\mathcal{Q}$ is the total spin $S_{Z}$ along the field direction. Now consider the situation at zero temperature $(T)$ as a chemical potential $\mu$ conjugate to $\mathcal{Q}$ is varied [6]. We are interested in a $\mathrm{QCP}$ at which $\langle\mathcal{Q}\rangle$ has a non-analytic dependence on $\mu[7]$; by a

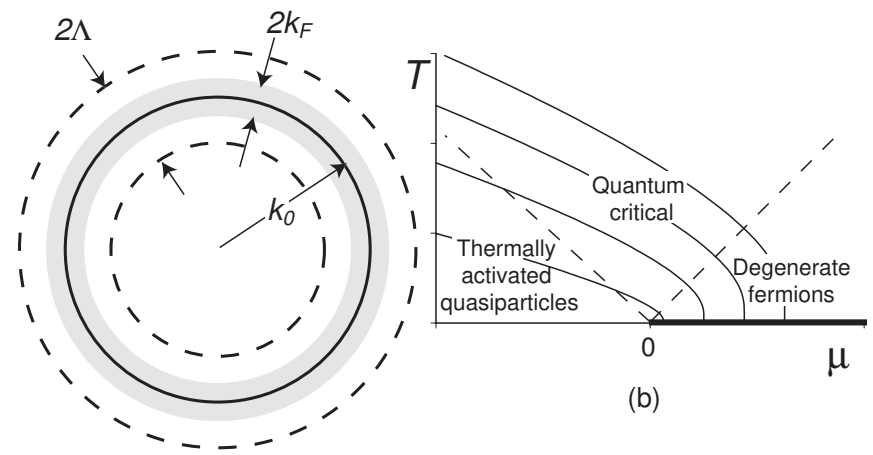

(a)

FIG. 1: Properties of $H$. (a) For $\mu<0$ the ground state of $H$ is the fermion vacuum, while for $\mu>0$ the ground state has fermions occupying the shaded region with $k_{0}-k_{F}<$ $k<k_{0}+k_{F} . \Lambda$ is a high momentum cutoff. (b) Crossover phase diagram: the dashed lines indicate crossovers between the labeled regimes, and the universal interaction applies in all three regimes. The full lines are contours of constant $\langle\mathcal{Q}\rangle$. In applications to paired fermion problems, all phases are superfluid, and the FFLO or "breached-pair" phases appear at low $T$ in the degenerate fermion regime. Note that for experiments at fixed $\langle\mathcal{Q}\rangle$ (as in cold atoms) there is a substantial intermediate range of $T$ in the quantum critical region, even though the ground state is in the degenerate fermion regime.

shift in $\mu$, we choose this QCP to be at $\mu=0$. Typically, $\langle\mathcal{Q}\rangle$ is independent of $\mu$ for $\mu<0$, and increases smoothly with $\mu$ for $\mu>0$.

We will describe the situation where for $\mu<0$ there is a gapped fermionic quasiparticle excitation which has minimum in its dispersion on a spherical shell of momentum $\mathbf{k}$ with $k=k_{0} \neq 0$ (the $k_{0}=0$ case is considered in Ref. [7). At $\mu=0$, the minimum energy of this fermionic excitation vanishes, and the onset in $\langle\mathcal{Q}\rangle$ variation is accompanied by the appearance of two new Fermi surfaces at wavevectors below and above $k_{0}$ (see Fig. [1 $\mathrm{H}$ ). One of our primary results is that the interactions between low lying fermion excitations at wavevectors near $k=k_{0}$ are universal, and we will determine some of these interactions exactly.

It is worth noting here that solution of the conventional weak-coupling Bardeen-Cooper-Schrieffer (BCS) theory does indeed yield a minimum in the quasiparticle dis- 
persion at a $k_{0} \neq 0$. However, application to the unbalanced fermion case does not yield a QCP as described above: instead there is a strong first order transition from a state with $\langle\mathcal{Q}\rangle=0$ to a state with $\langle\mathcal{Q}\rangle \neq 0$ at some $\mu<0$ [8]; similar first-order transitions have also been obtained in recent mean-field analyses 9, 10, 11]. On the other hand, there is recent numerical evidence for a continuous transition 12], in a situation with strong bare interactions between the fermions and a renormalized quasiparticle dispersion minimum at a $k_{0} \neq 0$. We will find here that a continuous transition can be present for generic short-range interactions between the fermions. As is also the case for many other second-order critical points [13], long-wavelength sound-mode fluctuations of the compressible environment lead to marginally relevant flows and a likely weak fluctuation-induced first order transition [14]. Such a transition is best described in the renormalization group framework introduced here, and is distinct from that of the weak-coupling mean-field analyses. In all cases, the underlying second-order QCP remains important for understanding the full scope of the crossovers and transitions in the $\mu-T$ plane.

We label the low-lying fermionic excitations in the vicinity of $k=k_{0}$ by annihilation operators $c_{\mathbf{k}}$, so that $\mathcal{Q}=\sum_{\mathbf{k}} c_{\mathbf{k}}^{\dagger} c_{\mathbf{k}}$; note that the fermions do not carry any additional 'flavor' or 'spin' label associated with quantum numbers distinct from $\mathcal{Q}$, but it is not difficult to extend our analysis to include this. The universal quantum critical properties can then be described by the following simple Hamiltonian

$$
H=\sum_{\mathbf{k}}[\epsilon(k)-\mu] c_{\mathbf{k}}^{\dagger} c_{\mathbf{k}}+\frac{1}{A} \sum_{\mathbf{k}, \mathbf{k}^{\prime}, \mathbf{q}} V_{q} c_{\mathbf{k}+\mathbf{q}}^{\dagger} c_{\mathbf{k}^{\prime}-\mathbf{q}}^{\dagger} c_{\mathbf{k}^{\prime}} c_{\mathbf{k}},
$$

where $V_{q}$ is a generic two-body interaction; $A$ is the volume of the system, and the single-particle dispersion is

$$
\epsilon(k)=\left(k-k_{0}\right)^{2} /\left(2 m^{*}\right) .
$$

For repulsive interactions, $H$ has a QCP at $\mu=0$, where the fermion density increases continuously from zero as $\mu$ increases. We will determine the critical behavior of the system at $\mu=0$, and also study the properties of the system in the dilute limit $k_{F} \approx \sqrt{2 m^{*}|\mu|} \ll k_{0}$ (see Fig. 1 $\mathrm{l}$ ). In two dimensions (2D) we find this low-density phase is a crystalline phase with charge density wave (CDW) order; this corresponds to the Fulde-Ferrell-Larkin-Ovchinnikov (FFLO) state 15, 16] in the fermion pairing problem. On the other hand we find in 3D a uniform phase is stable against CDW instability when the interaction is sufficiently weak; this corresponds to the "breached pairing" phase [9].

The Quantum Critical Point - For simplicity we will focus mainly on $2 \mathrm{D}$, and briefly comment on the $3 \mathrm{D}$ case. Near the QCP at $\mu=0$, the important low-energy fermionic modes are those lie along a shell: $k_{0}-\Lambda<k<$
$k_{0}+\Lambda$, with the cutoff $\Lambda \ll k_{0}$; these are the modes we keep in our model. Thus the phase space structure and corresponding kinematic constraints of the present problem is quite similar to that of the Fermi liquid fixed point studied by Shankar [17] using momentum shell renormalization group (RG). There are, however, some important differences. First, the quadratic fermion dispersion (2) changes the scaling dimension of various operators. Second, the ground state at the QCP is known exactly; it is simply the fermion vacuum. This immediately leads to the exact fermion $T=0$ Green's function (which is not modified by interactions):

$$
G(\omega, \mathbf{k})=1 /[i \omega-\epsilon(k)] .
$$

The exact two fermion scattering vertices (or four-point functions) $\Gamma\left(\mathbf{k}_{1}, \omega_{1}, \mathbf{k}_{1}^{\prime}, \omega_{1}^{\prime} ; \mathbf{k}_{1}+\mathbf{q}, \omega_{1}+\omega, \mathbf{k}_{1}^{\prime}-\mathbf{q}, \omega_{1}^{\prime}-\omega\right)$ can be obtained simply by summing ladder diagrams. Here we need to divide the scattering vertices into two classes. (i) The total momentum of the two particles $|\mathbf{Q}|=\left|\mathbf{k}_{1}+\mathbf{k}_{1}^{\prime}\right| \gg \Lambda$; in this case kinematic constraints restrict the scattering processes with momentum transfer $|\mathbf{q}| \lesssim \Lambda$, i.e., only forward scattering is possible in this case [17]. (ii) $|\mathbf{Q}|=\left|\mathbf{k}_{1}+\mathbf{k}_{1}^{\prime}\right| \ll \Lambda$; in this case large angle scattering is possible, and these are the processes in the Cooper channel 17.

For case $(i)$ the one-loop correction to $\Gamma$ is

$$
\begin{aligned}
F^{(2)}(\theta, \Omega)= & -\int \frac{d^{2} \mathbf{q}}{(2 \pi)^{2}} \frac{[V(\theta)]^{2}}{-i \Omega+\frac{\left(\left|\mathbf{k}_{1}+\mathbf{q}\right|-k_{0}\right)^{2}+\left(\left|\mathbf{k}_{1}^{\prime}-\mathbf{q}\right|-k_{0}\right)^{2}}{2 m^{*}}} \\
& =-\frac{m^{*}[V(\theta)]^{2}}{2 \pi|\sin \theta|} \log \frac{\Lambda^{2}}{2 m^{*}|\Omega|}
\end{aligned}
$$

where $\Omega=\omega_{1}+\omega_{1}^{\prime}=\omega_{2}+\omega_{2}^{\prime}, \theta$ is the angle between $\mathbf{k}_{1}$ and $\mathbf{k}_{1}^{\prime}$, and $V(\theta)=V_{q=0}-V_{2 k_{0} \sin (\theta / 2)}$. The ladder sum is sum over a geometric series that yields:

$$
\Gamma=F(\theta, \Omega)=V(\theta) /\left[1+\frac{m^{*} V(\theta)}{2 \pi|\sin \theta|} \log \frac{\Lambda^{2}}{2 m^{*}|\Omega|}\right] .
$$

Clearly $F$ approaches a universal function in the lowenergy limit $\Omega \rightarrow 0$, in that it is independent of the bare interaction $V$, when it is repulsive.

For case $(i i)$, because $\mathbf{k}^{\prime} \approx-\mathbf{k}$, it is convenient to re-parameterize the vertex as $\Gamma\left(\mathbf{k}_{1}, \omega_{1},-\mathbf{k}_{1}+\mathbf{Q}, \Omega-\right.$ $\left.\omega_{1} ; \mathbf{k}_{2}, \omega_{2},-\mathbf{k}_{2}+\mathbf{Q},-\omega_{2}+\Omega\right)$, with $Q \ll \Lambda$, and decompose it into angular momentum channels: $\Gamma=$ $\sum_{n} U_{n}(Q, \Omega) e^{i n\left(\theta_{\mathbf{k}_{1}}-\theta_{\mathbf{k}_{2}}\right)}$. A similar ladder sum yields

$U_{n}(Q, \Omega)=V_{n} /\left\{1+V_{n} k_{0}\left[\sqrt{\frac{m^{*}}{4|\Omega|}} f\left(\frac{Q^{2}}{2 m^{*}|\Omega|}\right)-\frac{m^{*}}{\pi \Lambda}\right]\right\}$,

where $V_{n}=\frac{1}{2 \pi} \int d \theta e^{-i n \theta}[V(\pi-\theta)-V(\theta)]$ (thus $n$ is odd), and $f(x)$ is a scaling function with the following asymptotic behavior: $f(x \rightarrow 0) \rightarrow 1$, and $f(x \rightarrow \infty) \rightarrow$ $\sqrt{\frac{2}{\pi^{2} x}} \log (x)$. Again the vertex function takes a universal form in the limit $\Omega, Q \rightarrow 0$ for repulsive interactions. 
It is interesting to interpret the above results in the RG langauge. The exact result in Eq. (3) indicates that the dynamic exponent $z=2$, the correlation length exponent $\nu=1 / 2$, and no fermion anomalous dimension $\eta=0$. However, the presence of the dimensionful scale $k_{0}$ means that, strictly speaking, there is no true scaleinvariant fixed point, and scaling arguments are of limited utility. Nevertheless, it is possible to write down RG equations and assign scaling dimensions for observables. The powers of $k_{0}$ associated with an observable cannot be predicted a priori, and explicit calculation is required. For forward scattering we obtain the RG equation

$$
\frac{d V(\theta)}{d \log s}=-\frac{m^{*}}{\pi|\sin \theta|}[V(\theta)]^{2},
$$

where $s$ is the momentum re-scaling factor for $\Lambda$. This implies forward scattering is marginally irrelevant/relevant for repulsive/attractive interactions. The solution of Eq. (77) also yields the universal structure in Eq. (5). In the Cooper channel, we have

$$
\frac{d V_{n}}{d \log s}=-\frac{m^{*} k_{0}}{\pi \Lambda(s)} V_{n}^{2} .
$$

We can remove the explicit dependence of the $\beta$ function on $\Lambda(s)$ by defining $\tilde{V}_{n}=V_{n} / \Lambda(s)$, and the flow equation for $\tilde{V}_{n}$ then takes the form

$$
\frac{d \tilde{V}_{n}}{d \log s}=\tilde{V}_{n}-\frac{m^{*} k_{0}}{\pi} \tilde{V}_{n}^{2}
$$

We thus find $\tilde{V}_{n}$ flows to a fixed point value of $\frac{\pi}{m^{*} k_{0}}$ if it is repulsive initially. The physical $V_{n}$ is then obtained by rescaling with the observed frequency scale, a conclusion consistent with Eq. (6).

The universal scattering vertices lead to a universal $T$ dependence of the quasiparticle scattering rate (or inverse lifetime), $1 / \tau$, in the quantum-critical regime of Fig. 1b. The large density of low energy states associated with the quadratic quasiparticle dispersion leads to a short lifetime, and its value has to be determined by computing the scattering rate into quasiparticle states which have been self-consistently broadened. A standard computation of the scattering cross-section between a quasiparticle on the Fermi surface and pre-existing thermally excited quasiparticle to order $\Gamma^{2}$ leads to the estimate

$$
\frac{1}{\tau_{2 D}} \sim T\left(m^{*} \Gamma\right)^{2}\left(k_{0}^{2} / m^{*}\right)^{1 / 2}\left[\operatorname{Max}\left(T, 1 / \tau_{2 D}\right)\right]^{-1 / 2} .
$$

The only self-consistent solution of this is

$$
\frac{1}{\tau_{2 D}}=\frac{C_{2} T^{2 / 3}\left(k_{0}^{2} / m^{*}\right)^{1 / 3}}{\left|\log \frac{\Lambda^{2}}{2 m^{*} T}\right|^{4 / 3}}
$$

where $C_{2}$ is a universal constant of order unity. The scattering rate is universal in the sense that it is independent of the bare interaction.
If the interaction is attractive, $\Gamma$ diverges at low energy in all channels, and the strongest divergence is in the Cooper channel, signaling the formation of bound states before the QCP at $\mu=0$ is reached; such bound states appear at $\mu=-V_{\ell}^{2} k_{0}^{2} m^{*} / 8$, where $\ell$ is the angular momentum channel with the strongest pairing interaction (i.e., $V_{l}$ being the most negative).

Many of these results can be generalized to the 3D case with very minor modifications. The most important difference between $2 \mathrm{D}$ and $3 \mathrm{D}$ is in case (i), the forward scattering channel. While in 2D the kinematic constraint only allows for small momentum transfer (or forward scattering), in 3D the scattering process allows for a rotation of the two momenta along the direction of $\mathbf{Q}$ while preserving the angle between the two momenta [17], i.e. $\mathbf{k}_{1} \cdot \mathbf{k}_{1}^{\prime} \approx \mathbf{k}_{2} \cdot \mathbf{k}_{1}^{\prime}$ or $\theta_{\mathbf{k}_{1}, \mathbf{k}_{1}^{\prime}} \approx \theta_{\mathbf{k}_{2}, \mathbf{k}_{2}^{\prime}} \approx \theta$. The angle of rotation $\phi=\phi_{\mathbf{k}_{1}}-\phi_{\mathbf{k}_{2}}$ ranges from 0 to $2 \pi$. Thus in $3 \mathrm{D}$ the scattering vertex is parameterized as $F(\theta, \phi, \Omega)$. Performing a Fourier transform with respect to $\phi: F(\theta, \phi, \Omega)=\sum_{n} F_{n}(\theta, \Omega) e^{i n \phi}$, and performing the ladder sum yields

$$
F_{n}(\theta, \Omega)=V_{n}(\theta) /\left[1+\frac{m^{*} k_{0} V_{n}(\theta)}{4 \pi|\cos (\theta / 2)|} \log \frac{\Lambda^{2}}{2 m^{*}|\Omega|}\right],
$$

where

$$
V_{n}(\theta)=\frac{1}{2 \pi} \int_{0}^{2 \pi} d \phi\left(V_{q(\theta, \phi)}-V_{q(\theta, \pi-\phi)}\right) e^{-i n \phi}
$$

with $q(\theta, \phi)=2 k_{0} \sin (\theta / 2) \sin (\phi / 2)$. While $F_{n}(\theta, \Omega)$ approaches a universal function that does not depend on the bare interaction in the low energy limit, generically this is not the case for $F(\theta, \phi, \Omega)$. This is because $F(\theta, \phi, \Omega)$ receives contributions from all channels, and the energy scale below which $F_{n}(\theta, \Omega)$ approaches the universal function depends on $n$; this energy scale approaches zero as $n \rightarrow \infty$. As a consequence the quasiparticle scattering is nonuniversal in $3 \mathrm{D}$; a straightforward calculation similar to the $2 \mathrm{D}$ case finds that it is linear in $T$ with logarithmic corrections whose form depends on the details of the bare interaction.

The Low-density Phase - We now consider the phase with a small $\mu$ or fermion density $\rho$. In the absence of interaction, fermions occupy a thin "Fermi shell" with $k_{0}-k_{F}<k<k_{0}+k_{F}$, where the "Fermi wavevector" $k_{F}=\pi \rho / k_{0}$ in $2 \mathrm{D}$ and $\pi^{2} \rho / k_{0}^{2}$ in $3 \mathrm{D}$ is the half thickness of the shell (see Fig (1)). In the following we show that this Fermi shell state has a strong tendency toward charge density wave (CDW) ordering, as reflected by enhanced static density susceptibilities at certain wavevectors; the enhancement is due to the fact that the "Fermi velocity" $v_{F}=k_{F} / m^{*}$ vanishes as $k_{F} \rightarrow 0$, but the size of the Fermi surface remains finite and of order $k_{0}^{d-1}$. We will show that in $2 \mathrm{D}$ this enhancement is strong enough to render the system unstable against crystallization in the low-density limit, in the presence of a weak repulsive interactions between the fermions. 
The strongest enhancement of static density susceptibility for non-interacting fermions, $\chi^{0}(Q)$, is for $Q \approx 2 k_{F}$, a nesting vector that connects the inner and outer Fermi surfaces that enclose the Fermi shell. A straightforward calculation for $Q \ll k_{0}$ yields

$$
\chi_{2 D}^{0}(Q)=\frac{2 m^{*} k_{0}}{k_{F}} K_{2 D}\left(\frac{Q}{2 k_{F}}\right),
$$

where the dimensionless scaling function

$$
K_{2 D}(x)=\frac{1}{16 \pi^{2} x} \int_{0}^{2 \pi} \frac{d \theta}{\cos \theta} \log \left|\frac{1+x \cos \theta}{1-x \cos \theta}\right| .
$$

The result is similar in $3 \mathrm{D}$ :

$$
\begin{aligned}
\chi_{3 D}^{0}(Q) & =\frac{2 m^{*} k_{0}^{2}}{k_{F}} K_{3 D}\left(\frac{Q}{2 k_{F}}\right), \\
K_{3 D}(x) & =\frac{1}{8 \pi^{2} x} \int_{0}^{1} d t \log \left|\frac{1+x t}{1-x t}\right| .
\end{aligned}
$$

Both $K_{2 D}(x)$ and $K_{3 D}(x)$ are sharply peaked at $x=1$. The prefactor $k_{0} / k_{F}$ strongly enhances $\chi_{0}$ near the QCP; this enhancement arises from the nearly perfect nesting between Fermi surfaces which are curved in the same direction.

The other nesting wave vectors are $Q_{ \pm}=2 k_{0} \pm 2 k_{F}$, which connect the opposite ends of the outer and inner Fermi surfaces respectively. We find

$$
\chi_{2 D}^{0}\left(Q_{ \pm}\right)=\frac{2 m^{*}}{(2 \pi)^{2}} \sqrt{\frac{k_{0}}{k_{F}}} \int_{0}^{\infty} d t \frac{\log \left(1+\frac{4}{t^{2}}\right)}{2+t^{2}}
$$

and $\chi_{3 D}^{0}\left(Q_{ \pm}\right)=m^{*} k_{0} / 32$. We find $\chi^{0}\left(Q_{ \pm}\right)$diverges in the low density limit in $2 \mathrm{D}$ while saturates in $3 \mathrm{D}$.

Now let us go beyond non-interacting fermions, and include the interactions computed earlier in a RPA-like theory of the susceptibility $\chi$. Naively one might expect repulsive interactions will immediately lead to a CDW instability at the wavevector $Q=2 k_{F}$ due to the divergent $\chi^{0}$ as $k_{F} \rightarrow 0$. However, there is a cancelation of interaction vertices in this limit for such small momentum transfer; as a consequence the strongest CDW instability is at larger wavevectors $Q_{ \pm}$, even though $\chi^{0}$ is less singular there. If we assume the $q$ dependence of $V_{q}$ is smooth over the scale of $2 k_{F}$, which is the case for short-range interactions, we can easily sum over the particle-hole bubble and ladder diagrams and obtain

$$
\chi(Q) \approx \frac{\chi^{0}(Q)}{1-\left(V_{q=0}-V_{Q}\right) \chi^{0}(Q)} .
$$

While $\chi^{0}(Q)$ is largest at $Q=2 k_{F}$, interaction does not immediately lead to an instability at this wavevector in the low density limit, as for generic short-range interactions we expect $V_{q=0}-V_{Q} \propto Q^{2}$ for small $Q$. We find instead that the strongest density instability is at $Q=Q_{ \pm}$, where the interaction effects are stronger. In particular, as long as $V_{q=0}-V_{2 k_{0}}>0$, in $2 \mathrm{D} \chi_{2 D}\left(Q_{ \pm}\right)$always diverges in the low-density limit $k_{F} \rightarrow 0$, suggesting that the fermions form a charge density wave or crystal state in the low density limit. Physically the CDW instability at wavevectors that are almost independent of density in the low-density limit can be understood in the following manner. In our model the low-energy single fermion states are those with $k \approx k_{0}$; thus localized states constructed from them must be oscillatory with a characteristic wavevector $2 k_{0}$, similar to the Friedel oscillation; this leads to strong CDW instability at such wavevectors. We further note that in the low-density limit the instability has equal strength for $Q_{ \pm}=2 k_{0} \pm 2 k_{F}$; as a consequence density modulations with characteristic wavevectors $O\left(k_{F}\right)$ will be present due to interference. We expect their magnitudes to be very large as $\chi$ is very strongly enhanced there for small $k_{F} / k_{0}$. On the other hand the in $3 \mathrm{D}$ uniform state appears to be stable against weak interaction, due to the fact that $\chi_{3 D}^{0}\left(Q_{ \pm}\right)$is finite.

To summarize, we have presented a new perspective on the physics of paired fermion systems, for the case in which the fermion densities are unbalanced. It has recently become possible to study such ultracold atom systems [3], while condensed matter systems have been under investigation for some time [4, 5]. Essentially all previous analyses have been carried out in the framework of extensions to BCS theory, seeking to minimize the free energy with respect to variations in the magnitude and spatial dependence of the order parameter. We have argued here that a more rigorous approach is to focus on the interactions between the renormalized quasiparticles as the system approaches a transition to a gapless phase. This allowed us to make a number of controlled predictions on the influence of strong interactions on the quasiparticle dynamics and the structure of proximate phases in the $\mu-T$ phase diagram.

This work was supported by NSF grants DMR-0225698 (KY) and DMR-0537077 (SS).

* On leave from Department of Physics, Florida State University, Tallahassee, FL 32306.

[1] P. Coleman and A. J. Schofield, Nature 433, 226 (2005).

[2] M. Greiner, O. Mandel, T. Esslinger, T. W. Hänsch, and I. Bloch, Nature 415, 39 (2002).

[3] M. W. Zwierlein et al., Science 311, 492 (2006); G. B. Partridge et al., Science 311, 503 (2006).

[4] H. A. Radovan et al., Nature 425, 51 (2003); A. Bianchi et al., Phys. Rev. Lett. 91, 187004 (2003).

[5] For a review, see R. Casalbuoni. and G. Nardulli, Rev. Mod. Phys. 76, 263 (2004).

[6] The electronic superconductors are naturally at fixed $\mu$ (i.e. applied field), while the cold atoms are usually studied at fixed $\langle\mathcal{Q}\rangle$; nevertheless, even for the latter it is useful to first consider fixed $\mu$, and later map the behavior along contours of fixed $\langle\mathcal{Q}\rangle$ - see Fig 1 b. 
[7] S. Sachdev, Quantum Phase Transitions, Cambridge University Press, Cambridge, 1999.

[8] A. M. Clogston, Phys. Rev. Lett. 3, 266 (1962).

[9] W. V. Liu and F. Wilczek, Phys. Rev. Lett. 90, 047002 (2003); M. M. Forbes, E. Gubankova, W. V. Liu, and F. Wilczek, Phys. Rev. Lett. 94, 017001 (2005).

[10] D. T. Son and M. A. Stephanov, cond-mat/0507586

[11] D. E. Sheehy and L. Radzihovsky, cond-mat/0508430

[12] J. Carlson and S. Reddy, Phys. Rev. Lett. 95, 060401 (2005).
[13] E. Frey and L. Balents, Phys. Rev. B 55, 1050 (1997); D. J. Bergman and B. I. Halperin, Phys. Rev. B 13, 2145 (1976).

[14] S. Sachdev and K. Yang, Phys. Rev. B 73, 174504 (2006).

[15] P. Fulde and R. A. Ferrell, Phys. Rev. 135, A550 (1964).

[16] A. I. Larkin and Yu. N. Ovchinnikov, Sov. Phys. JETP 20, 762 (1965).

[17] R. Shankar, Rev. Mod. Phys. 66, 129 (1994). 